\title{
Ca Chelators and Membrane Transport: "Geometric" Considerations
}

\author{
Ronald F. Abercrombie ${ }^{\mathrm{a}, *}$ and James E. Moore ${ }^{\mathrm{b}}$ \\ Departments of Physiology ${ }^{a}$ and Pediatrics ${ }^{b}$, Emory University School of Medicine, Atlanta, GA, 30322, USA
}

\begin{abstract}
We examine theoretically the ability of diffusible cytoplasmic carriers to facilitate delivery of substrate to sites within biological membranes. Two processes are analyzed. In one, delivery of Ca to a site on a membrane surface is examined. As a contrasting example, chelator-supported diffusion to a uniform absorbing surface is considered.
\end{abstract}

\section{INTRODUCTION}

Intracellular $\mathrm{Ca}$ exerts control over a range of cellular responses. Maintaining a low basal intracellular $\mathrm{Ca}$ ion concentration provides the background for calcium signaling, and this requires transport via pumps into organelles and across the plasma membrane. We consider whether the presence of mobile intracellular calcium chelators aids the transport process and examine how "geometric" considerations enter the analysis by contrasting a regime of small transport sites to one in which absorption takes place at any location on a surface.

Calcium and diffusion to transport sites: possible role for chelated Ca ions. In its most basic representation, the $\mathrm{Ca}$ ion activity relates to the frequency of strikes that the ions make against a site. If we imagine a small entry site residing on the surface of a calcium pump, then the local calcium activity would be proportional to the frequency of $\mathrm{Ca}$ ion strikes against this site. The central question becomes the following: "If calcium is available on mobile chelators, could the local $\mathrm{Ca}$ ion activity at the site rise above that expected when no mobile chelators are present?" In other words, with a favorable local electrostatic or ionic environment around a binding site, would the presence of calcium chelators influence the frequency with which these ions strike the site?

To formulate this question we follow a treatment of Decker and Levitt [1], originally derived for hydrogen ion flux through pores, which finds its basis in a treatise of Smoluchowski [2]. In the development of these equations, it is generally assumed that the target absorbs each ion that strikes it, providing a mathematical boundary condition of zero ion concentration at the target surface. A zero ion concentration may seem a stringent physical constraint at the entry site of $\mathrm{Ca}$ ions. However, an equivalent assumption which contains the same mathematical boundary condition is that each $\mathrm{Ca}$ ion may strike this site only once and that an approach and strike of one ion, followed by its return to the medium, would not interfere with that of another. This assumption likely becomes more realistic in the low calcium concentration limit. We therefore consider unidirectional flux toward a site, without the second-order complications of ion-ion interference, and thus we seek a quantitative description of diffusion-limited strike frequency that would apply at the low concentration limit.

*Address correspondence to this author at the Department of Physiology, Emory University School of Medicine, Atlanta, GA, 30322, USA; Tel: (404) 727-7425; Fax: (404) 727-2648;

E-mail: Ronald.abercrombie@emory.edu

\section{MATHEMATICS OF DIFFUSION TO DISCRETE SITES}

The diffusion of calcium ions of concentration $[\mathrm{Ca}]$ surrounding the surface of a "dome" representing a calcium entry site (a spatially defined target) must satisfy the diffusion-reaction equation, given here in spherical coordinates:

$$
\frac{\partial[C a]}{\partial t}=D_{C a} \frac{1}{r^{2}} \frac{\partial}{\partial r}\left(r^{2} \frac{\partial[C a]}{\partial r}\right)+F
$$

We define $F$ as the net feed rate from the buffered pool. This term represents an excess delivery (above diffusion) of $\mathrm{Ca}$ ions which dissociate from a nearby buffer. $F=0$ for the case of simple diffusion without a calcium chelator. $D_{C a}$ is the free calcium diffusion coefficient; $r$ is the distance from the center of a dome representing the pump's calcium inlet site; and $t$ is time. We give solutions of this equation for the steady state, $\frac{\partial[\mathrm{Ca}]}{\partial t}=0$, thus representing an average behavior over time.

For a uniform concentration of chelator $[B]_{b}$ and chelated $\mathrm{Ca}[\mathrm{BCa}]_{b}$ and a fixed calcium concentration $[\mathrm{Ca}]_{b}$ in the bulk solution, the term $F$ in equation (1), representing the extra delivery to the site via the chelator (net feed rate), may be derived from $F=k_{o f f}[B C a]_{b}-k_{o n}[B]_{b} \cdot[C a]$ by substituting the equilibrium expression for $k_{\text {on }}$ within the bulk solution as $k_{o n}=\frac{k_{o f f}[B C a]_{b}}{[B]_{b}[C a]_{b}}$,

where $k_{o f f}$ is the rate constant of Ca-chelator dissociation and $k_{o n}$ is that of association.

$F$ would then be represented in the following form:

$$
F=k_{\text {off }}[B C a]_{b}\left(\frac{[C a]_{b}-[C a]}{[C a]_{b}}\right)
$$

In biological solutions, $k_{\text {off }}$ typically would be in the range of $1-100 \mathrm{~s}^{-1}$ for chelators such as BAPTA or EGTA at neutral $\mathrm{pH}$. The terms $[\mathrm{BCa}]_{b}$, the buffered calcium concentration, and $[B]_{b}$, the free buffer concentration, represent these values in bulk solution as well as near the site. These concentrations would be in the millimolar range, and nearly uniform around the site if the local dwell time of the Cachelator were much less than the reciprocal of the off-rate constant, $1 / k_{\text {off }}(\sim 0.01$ to $1 \mathrm{~s})$. A dwell time, $\Delta \mathrm{t}_{\mathrm{d}}$, given by $\Delta \mathrm{t}_{\mathrm{d}}$ $=(\Delta x)^{2} / D_{B} \approx 10^{-9} \mathrm{~s}$ for values of $\Delta x$ in the $\mathrm{nm}$ range, and $D_{B}$ 
$\sim 10^{-5} \mathrm{~cm}^{2} \mathrm{~s}^{-1}$, ordinarily would satisfy this requirement. $\left(D_{B}\right.$ represents the chelator diffusion coefficient.)

The average calcium concentration profile $[\mathrm{Ca}]$ (a function of $r$ ) may be obtained by solving the diffusion-reaction equation (1) with the appropriate boundary conditions: in the bulk, $[\mathrm{Ca}]=[\mathrm{Ca}]_{b}$; and, at the reaction site, $[\mathrm{Ca}]=0$ (used to estimate unidirectional flux or strike frequency). Assuming that $F=0$, the steady-state solution of equation (1) is:

$$
\frac{[\mathrm{Ca}]_{b}-[\mathrm{Ca}]}{[\mathrm{Ca}]_{b}}=\frac{r_{o}}{r},\left(r \geq r_{o}\right)
$$

where $r_{o}$ is the radius of a dome representing the Ca site.

Assuming that a Ca buffer is present, with $F$ defined by equation (2), the steady-state solution for $[\mathrm{Ca}]$ from equation (1) is:

$$
\frac{[C a]_{b}-[C a]}{[C a]_{b}}=\frac{r_{o}}{r} \exp \left(\frac{r_{o}-r}{\lambda}\right),\left(r \geq r_{o}\right)
$$

with the constant $\lambda$ explicitly defined in equation (8). That this expression solves the differential equation (1) under the definition of $\mathrm{F}$ given in equation (2) can be confirmed by substitution of $[\mathrm{Ca}]$ from equation (4) into equation (1).

Taking the simpler case of no Ca chelators, i.e., $F=0$, with the $[\mathrm{Ca}]$ profile given as in equation (3), and assuming the site to be represented by a hemispherical dome of radius $r_{o}$, the standard expression can be derived for the steady-state diffusion-limited strike frequency $v$. Flux is determined from the Ca diffusion coefficient and the slope of the concentration profile and is converted to particles per second, $v$, by multiplying the target area and Avogadro's number.

$$
v=\left.D_{C a} \cdot 2 \pi r_{o}^{2} N \frac{\partial[C a]}{\partial r}\right|_{r=r_{o}}
$$

or

$$
v=D_{C a} \cdot[C a]_{b} \cdot 2 \pi r_{o} N,
$$

where $N$ is Avogadro's number, $r_{o}$ is in $\mathrm{cm}, D_{C a}$ is the free $\mathrm{Ca}$ diffusion coefficient $\left(\mathrm{cm}^{2} \mathrm{~s}^{-1}\right)$, and $[C a]_{b}$ is in units of moles $\mathrm{cm}^{-3}$.

In the presence of a Ca-releasing chelator, the expression similarly derived (from equation 4) for the frequency of strikes becomes (see, for example, [1])

$$
v=D_{C a} \cdot[C a]_{b} \cdot 2 \pi r_{o} N\left(1+r_{o} / \lambda\right),
$$

with $\lambda$ given by

$$
\lambda=\sqrt{\frac{[C a]_{b} \cdot D_{C a}}{[B C a]_{b} \cdot k_{o f f}}}
$$

$[B C a]_{b}$ must have the same units as $[\mathrm{Ca}]_{b}$ and $\lambda$ has units of $\mathrm{cm}$, if $D_{C a}$ has units of $\mathrm{cm}^{2} \mathrm{~s}^{-1}$.

Equations (6) and (7) are central to this discussion and follow directly from equations (3) and (4), respectively. These results should be valid as long as no interference takes place among striking and exiting $\mathrm{Ca}$ ions at the site, each approaching $\mathrm{Ca}$ ion strikes the site only once, and a uniform concentration of chelated Ca surrounds the site. If these conditions do not prevail, then (7) may represent a lower limit as discussed later. Equations (7) and (8) thus provide a basis for estimating the effects of the variables $[C a]_{b}, k_{o f f}, D_{C a},[B C a]_{b}$, and $r_{o}$ on $\mathrm{Ca}^{2+}$ delivery to a hypothetical target. They illustrate that a $\mathrm{Ca}$ ion striking the site may come from one of two possible sources: a free $\mathrm{Ca}$ ion in solution, or a calcium ion that is released from a chelator molecule as it migrates sufficiently close to the site.

As shown in equation (7), the relative size of the second component, or the chelator contribution, is given by the ratio of the capture site radius $r_{o}$ compared to the parameter $\lambda$. For the case of $\mathrm{Ca}$ transport, $\lambda$ would be defined in a region about which we have limited knowledge. If we use the known values of the free Ca diffusion coeffieient $D_{C a}\left(\sim 10^{-5} \mathrm{~cm}^{2} \mathrm{~s}^{-1}\right), k_{\text {off }}$ in free solution for chelators such as EGTA or BAPTA $\left(\sim 1 \mathrm{~s}^{-1}-100 \mathrm{~s}^{-1}\right)$, and typical values for $[B C a]_{b}\left(\sim 10^{-3} \mathrm{M}\right)$ and $[C a]_{b}\left(\sim 10^{-7} \mathrm{M}\right)$, then the parameter $\lambda$ would be in the $\sim 30-$ $300 \mathrm{~nm}$ range, larger than any reasonable estimate of a target size $r_{o}(\sim 0.1-1 \mathrm{~nm})$. Thus, there would be little significant contribution of $\mathrm{Ca}$ ions released by chelators onto a small site, assuming that these reside in free solution of biological media. If $\mathrm{Ca}$ chelators were to contribute to transport, then either they must have very fast $k_{\text {off }}$ rates or an unusual microscopic environment must prevail near the capture site. Thus, (a) the Ca off-rate, $k_{\text {off }}$, could be much greater at the site, (b) the diffusion coefficient of free calcium, $D_{C a}$, could be much lower than in bulk solution, (c) the effective concentration of chelated calcium at the site, $[B C a]$, could be greater than in bulk solution, or perhaps (d) the size of the capture site, $r_{o}$, could be larger than we would predict. If $r_{o}$ were large compared to $\lambda$ (for example if buffers are present in large concentrations or have a high $k_{\text {off }}$ ), then according to equation (7), chelators would aid solute movement to the capture site. A capture site for $\mathrm{Ca}$ transport, $r_{o}$, is expected to be small $(\sim \mathrm{nm})$, however, and buffer facilitation of transport is not expected, assuming free solution values for $[C a]_{b}, D_{C a}$, $[B C a]_{b}$, and values for $k_{\text {off }}$ of $\sim 1 \mathrm{~s}^{-1}-100 \mathrm{~s}^{-1}$.

\section{DIFFUSION OF MEMBRANE-PERMEANT SUB- STANCES TO A PLANAR SURFACE}

The previous calculations considered three-dimensional diffusion toward small sites. As a comparison, we consider a calculation for diffusion to, and absorption at, any location on a planar surface. Though not an appropriate model for $\mathrm{Ca}$ transport, this might apply to substrates such as oxygen or carbon dioxide, which are capable of unrestricted crossing of a lipid membrane. We nevertheless consider the diffusible cytoplasmic carrier for the membrane-permeant substrate (oxyhemoglobin or bicarbonate) as a "chelator/carrier".

For absorption at any location on a planar membrane, the problem reduces to one dimension as expressed in equation (9):

$$
\frac{\partial[C]}{\partial t}=D_{C} \frac{\partial^{2}[C]}{\partial x^{2}}+F
$$

$D_{C}$ and $[C]$ represent the diffusion coefficient and concentration of the membrane permeant (free) substrate and $x$ is the distance from the surface. With $F$ defined as before, the steady-state solution to equation (9) can be represented as 


$$
\frac{[C]}{[C]_{b}}=1-\exp (-x / \lambda),
$$

where $[C]_{b}$ represents the bulk substrate concentration, and $\lambda$ has its previous meaning. Here the introduction of the "feed" term " $F$ " in equation (9) changes the solution from what would have been a linear one (with $F=0$ ) to an exponential form, equation (10).

From equations (10) and (8), and $v=N \cdot$ area $\cdot D_{C} \cdot \frac{d C}{d x}$, the representation of strikes to an area of planar surface per second is given by $\frac{v}{\text { area }}=N \sqrt{D_{C}[C]_{b} \cdot[B C]_{b} k_{\text {off }}}$, which explicitly includes a chelator/carrier contribution in the term $[B C]_{b}$.

Another approach to providing a solution to equation (9) involves the explicit introduction of an unstirred layer and defining the distance over which a concentration gradient may exist close to the membrane surface. Here, the probability of a chelator contribution to the flux is related to the ratio of the size of the unstirred layer compared to $\lambda$ (defined in equation 8).

In a third and simpler approach, the diffusion to a "planar-like" surface may be approximated by simply increasing the size of the target $\left(r_{o}\right)$ in (7), corresponding to a larger region where the "chelated" molecules interact with a surface. Here, for example, the parameter $r_{o}$ could represent the radius of a vesicle, the surface of which is highly permeable to the substrate, $[C]_{b}$. Thus the entire vesicle would constitute a target. Though the size of the parameter $\lambda$ would depend on $[C]_{b}, k_{o f f}, D_{C}$, and $[B C]_{b}$ as given in an expression similar to that of equation (8), the size of " $r_{o}$ " for this process becomes as large as the vesicle, increasing the likelihood of chelator facilitation of flux of membrane-permeant substrates according to (7).

\section{MEASURED CHELATOR EFFECTS}

Is there evidence that $\mathrm{Ca}$ chelators facilitate $\mathrm{Ca}$ transport? Even though this transport must act through relatively small transport sites, the answer is, perhaps surprisingly, yes. Effects of chelators on calcium transport have been reported in studies of both plasma membrane (PM) and endoplasmic reticulum (ER) type Ca pumps. Most of these studies were done measuring $\mathrm{Ca}$ flux with ${ }^{45} \mathrm{Ca}$, and all were done using isolated membrane vesicles. For example, in 1979, Sarkadi, Shubert, and Gardos [3] demonstrated that Ca pumps of inside-out vesicles of erythrocyte membranes had a higher apparent $\mathrm{Ca}$ affinity when EGTA was present. In 1982, Berman [4] isolated vesicles of sarcoplasmic reticulum (SR) and, using calcium electrodes, detected SR calcium uptake stimulated by the presence of EGTA. He showed that the Ca pump of SR vesicles had a greater apparent $\mathrm{Ca}$ affinity when $50 \mu \mathrm{M}$ EGTA was included in the uptake media. It is well known that calmodulin, an intracellular messenger that binds $\mathrm{Ca}$, activates the PM Ca pump at a specific Ca-calmodulin receptor site ([5], [6]). Timmermans et al. [7] concluded that other calcium chelators (EGTA, calbindin-D28, and parvalbumin) increase the PM pump activity by another, fully distinct, mechanism: that of increasing the "free $\mathrm{Ca}$ in the immediate vicinity of the Ca pump". Enhancement of Ca flux by calbindin-D28 or EGTA was additive and independent of that caused by calmodulin. Thus, EGTA and calbindin had actions that were different from the regulatory cofactor, calmodulin. Calbindin, the native vitamin-D dependent intracellular Ca-binding protein, helps to restore the body's calcium when $\mathrm{Ca}$ stores are depleted [8]. Chelator-assisted Ca delivery to plasma membrane pump inlet sites could be a mechanism of calbindin's action in maintaining a cell's and the organism's Ca homeostasis. In microsome (ER) preparations from brain, elevated $\mathrm{Ca}$ transport affinity was found in the presence of chelators, including EGTA, BAPTA, and BAPTA derivatives [9].

\section{THE LOCAL ENVIRONMENT}

What might be the local environment in which $\mathrm{Ca}$ and $\mathrm{Ca}$ chelators encounter $\mathrm{Ca}$ transport sites? Before the crystal structure of the SERCA pump was determined [10,11], this question could not have been satisfactorily addressed. Most might have predicted $\mathrm{Ca}$ entry via the hydrophilic regions of the pump protein that extend into the cytoplasm. The location of the $\mathrm{Ca}$ entry site, at least according to one of these crystal structures, is within a region that would be partly surrounded by hydrophobic surfaces, i.e., a region that would likely be adjacent to, or perhaps partially submerged within, the lipid bilayer. (This may be seen in supplementary material given by Toyoshima and Nomura [11]; their figure is reproduced with permission). Thus, a possible pathway for $\mathrm{Ca}$ entry begins on the cytoplasmic surface near the phospholipid bilayer; the access path may skirt the lipid interface, and then follow onto an opening and $\mathrm{Ca}$ binding site deeper

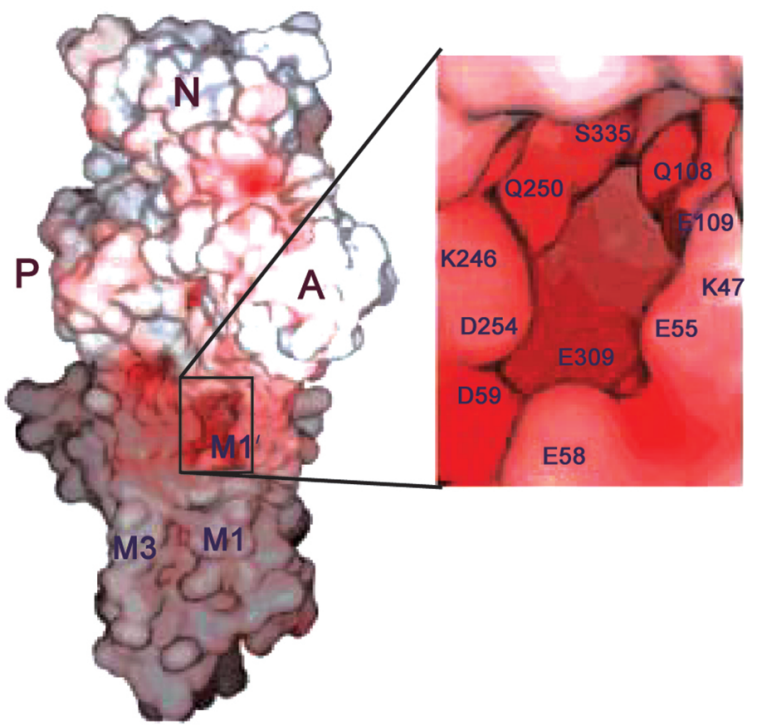

This Figure is taken with permission from the supplementary material of Toyoshima and Nomura [11]. A water-accessible channel leading to the $\mathrm{Ca}$ binding site within the protein is shown in the enlarged view of the inset to the right. Although the inlet channel itself has a predominantly negative charge (red in the color image) at its surface (to accommodate the positively charged calcium ion), this opening originates near the uncharged (hydrophobic) region of the molecule (grey in the color image), which would be embedded in the lipid of the bilayer membrane. "P", "N", and "A" domains are hydrophilic (cytoplasmic) domains and are generally positively charged (white); M1 and M3 represent portions of transmembrane alpha helices. The lettered symbols in the enlarged inset to the right represent identified amino acids of the inlet channel and are near the $\mathrm{Ca}$ binding site deeper within the pump protein. 
within the pump's structure. It is conceivable that accessory molecules (such as $\mathrm{Ca}$ chelators) might ease $\mathrm{Ca}^{2+}$ delivery across a partially hydrophobic environment helping provide access to the $\mathrm{Ca}$ inlet site. Chelators could thus act as local chaperones. In such a unique local environment, the parameter $\lambda$ would be smaller and the second term in equation (7), i.e., $r_{o} / \lambda$, larger than would be expected in free solution.

\section{SUMMARY AND CONCLUSIONS}

Two types of chelator effects appear at Ca pumps: a wellstudied specific effect caused by calmodulin's interaction with a calmodulin receptor site on plasma membrane (PM) type pumps, and a less well-studied, and theoretically unexpected, non-specific effect caused by a number of different $\mathrm{Ca}$ chelators on both the PM and ER type pumps. The nonspecific effect suggests the presence of an atypical microscopic environment surrounding the $\mathrm{Ca}$ inlet site that could alter a "chelator dwell time" or Ca off-rate.

For detecting the chelator effects that have been reported, experiments were always done using isolated membrane vesicles. If one accepts that native cellular $\mathrm{Ca}$ chelators function to facilitate Ca delivery to pump sites, it is not surprising that only when membranes have been stripped of their natural chelators by the isolation procedures of membrane preparation might one expect to identify a role of an exogenous chelator, as added synthetic or natural chelators replace those lost from the intact system. Ca delivery to its inlet site may take advantage of an interaction of low specificity between native $\mathrm{Ca}$ chelators and an unusual local environment at the intake site. This design could have been evolution's solution for two potentially conflicting requirements: maintaining a relatively low background of free intracellular calcium (for signaling purposes), but preserving the ability to rapidly transport large amounts of $\mathrm{Ca}$ across cellular membranes to quickly redistribute this ion.

\section{REFERENCES}

[1] Decker ER, Levitt DG. Use of weak acids to determine the bulk diffusion limitation of $\mathrm{H}^{+}$ion conductance through the gramicidin channel. Biophys J 1988; 53(1): 25-32.

[2] Smoluchowski MV. Drei Vortrage ueber Diffusion, Brownsche Molekularbewegung und Koagulation von Kolliodteilchen. Phys Z 1916; 17: 557-99.

[3] Sarkadi B, Schubert A, Gardos G. Effects of calcium-EGTA buffers on active calcium transport in inside-out red cell membrane vesicles. Experientia 1979; 35(8): 1045-7.

[4] Berman MC. Stimulation of calcium transport of sarcoplasmic reticulum vesicles by the calcium complex of ethylene glycol bis( $\beta$ aminoethyl ether)-N,N',-tetraacetic acid. J Biol Chem 1982; 257(4): 1953-7.

[5] Carafoli E, Niggli V, Malmstrom K, Caroni P. Calmodulin in natural and reconstituted calcium transporting systems. Ann N Y Acad Sci 1980; 356: 258-66.

[6] Niggli V, Adunyah ES, Penniston JT, Carafoli E. Purified $\left(\mathrm{Ca}^{2+}-\right.$ $\mathrm{Mg}^{2+}$ )-ATPase of the erythrocyte membrane. Reconstitution and effect of calmodulin and phospholipids. J Biol Chem 1981; 256(1): 395-401.

[7] Timmermans JA, Bindels RJ, van Os CH. Stimulation of plasma membrane $\mathrm{Ca}^{2+}$ pump by calbindin-D28k and calmodulin is additive in EGTA-free solutions. J Nutr 1995; 125 (7 Suppl): 1981S-6S.

[8] Feher JJ, Fullmer CS, Wasserman RH. Role of facilitated diffusion of calcium by calbindin in intestinal calcium absorption. Am J Physiol 1992; 262(2 Pt 1): C517-26.

[9] Moore, JE, Abercrombie RF. Calcium chelators enhance ${ }^{45} \mathrm{Ca}$ accumulation in permeablized synaptosomes and in microsomes. Am J Physiol 1996; 270(2 Pt 1): C628-35.

[10] Toyoshima C, Nakasako M, Nomura H, Ogawa H. Crystal structure of the calcium pump of sarcoplasmic reticulum at $2.6 \mathrm{~A}$ resolution. Nature 2000; 405(6787): 647-55.

[11] Toyoshima C, Nomura H. Structural changes in the calcium pump accompanying the dissociation of calcium. Nature 2002; 418(6898): 605-11. 